\title{
Aprendizaje cooperativo en los programas de entrenamiento de memoria multifacto- rial para personas mayores
}

Cooperative learning in multifactorial memory training programs for the elderly

Cristina Vidal-Martí

e-mail: cristinavidal@ub.edu

Universitat de Barcelona. España

\section{Resumen}

Los programas de entrenamiento de la memoria son una de las intervenciones cognitivas de carácter preventivo, dirigidas a personas mayores sin deterioro cognitivo. En la actualidad, hay una alta variedad de programas que difieren en su estructuración, orientación, organización y en aspectos educativos. Este artículo se centra en los programas de entrenamiento de memoria de tipo multifactorial. Una propuesta de intervención diseñada para trabajar procesos, estrategias y técnicas con el fin de estimular los distintos factores implicados en el funcionamiento de la memoria y los diferentes dominios cognitivos. El objetivo de este artículo es valorar los beneficios del aprendizaje cooperativo como metodología educativa aplicada a los programas de entrenamiento de la memoria. Esta metodología educativa que, en la actualidad, solo se implementa en los contextos educativos de tipo formal, puede favorecer un aprendizaje más inclusivo, duradero, significativo y la promoción del desarrollo de habilidades y técnicas relacionales. En resumen, contribuir a una mejora educativa de los programas de entrenamiento de la memoria. Las condiciones y los elementos diferenciales del aprendizaje cooperativo pueden ser características a aplicar en los programas de entrenamiento de la memoria, para favorecer el proceso de aprendizaje y adquirir habilidades que fomenten la cooperación y la ayuda mutua entre los participantes. Este artículo analiza estas características y propone su adaptación en los programas de entrenamiento de la memoria. En definitiva, el aprendizaje cooperativo es una buena metodología educativa para implementar en los programas de entrenamiento de la memoria.

Palabras clave: aprendizaje cooperativo; equipo, entrenamiento; memoria; personas mayores.

\begin{abstract}
Memory training programs are preventive cognitive interventions aimed at the elderly without cognitive impairment. Currently, there is a wide variety of programs that differ in their structure, orientation, organization and educational aspects. This article focuses on multi-factorial training programs. An intervention proposal designed to work: processes, strategies and techniques. Its purpose is to influence the different factors involved in the functioning of memory and the different cognitive domains. The objective of this article is to evaluate the benefits of cooperative learning as an educational methodology applied to memory training programs. Currently, this educational methodology, which is only implemented in formal educational contexts, can contribute to more inclusive, lasting, meaningful learning and promote the development of relational skills and techniques. In summary, contribute to an educational improvement of memory training programs. The conditions and differential elements of cooperative learning can be characteristics to be applied in memory training programs, to favour the learning process and acquire skills that foster cooperation and mutual help among participants. This article analyses these characteristics and proposes their adaptation in memory training programs. In summary, cooperative learning is a good educational learning methodology to implement in memory training programs.
\end{abstract}

Keywords: cooperative learning; equipment; training; memory; elderly.

Recibido / Received: 01-09-2019

Aceptado / Accepted: 30-03-2020

Publicación en línea / Published online: 29-04-2020

Cómo referenciar este artículo / How to reference this article:

Vidal-Martí, C. (2020). Aprendizaje cooperativo en los programas de entrenamiento de memoria multifactorial para personas mayores. Tendencias Pedagógicas, 36, pp. 154-163. doi: 10.15366/tp2020.36.12 


\section{Introducción}

El incremento notable de personas mayores en las sociedades occidentales y los cambios cognitivos, los cuales se empiezan a producir en la adultez, pero se visibilizan en la vejez, han facilitado la creación de servicios dirigidos a esta población para dar respuesta a sus necesidades; siendo uno de estos servicios los programas de estimulación cognitiva.

En sus inicios, estos programas eran de carácter rehabilitador, pero con los años, su orientación es más educativa. En la actualidad, son unas herramientas preventivas y de promoción de la salud (Hernández, 2005; Vidal-Martí, 2012b).

Las investigaciones centradas en la eficacia de dichos programas establecen que el entrenamiento tiene múltiples beneficios en las funciones cognitivas y es un efecto protector para prevenir el envejecimiento patológico (Novoa, Juárez, \& Nebot, 2008; Pérez et al., 2015).

A su vez, los procesos de innovación se focalizan en la metodología de la intervención (VidalMartí et al., 2013). Sin embargo, no se han encontrado intervenciones centradas en el método del proceso de aprendizaje-enseñanza y, por ese motivo, la finalidad de dicho manuscrito.

Este artículo valora la idoneidad de adaptar nuevas metodologías a dichos programas. Concretamente, se propone analizar y valorar los beneficios del aprendizaje cooperativo como metodología educativa para implementar en los programas de entrenamiento de la memoria de tipo multifactorial.

\section{Programas de entrenamiento de la memoria}

En los últimos años, las intervenciones cognitivas dirigidas a personas mayores de 60 años aumentaron de manera significativa. La preocupación por el deterioro cognitivo más el incremento de servicios y productos orientados a este grupo de edad contribuyó a aumentar el número de programas de intervención cognitiva (Novoa et al., 2008). Uno de dichos programas es el de entrenamiento de la memoria, conocidos popularmente como talleres de memoria.

Los primeros programas aparecieron alrededor de los años 70 . Se dirigían a personas con trastornos orgánicos cerebrales con el fin de estimular su capacidad cognitiva y promover la rehabilitación (Hernández, 2005). Sin embargo, con el paso del tiempo, se diversificaron; y en la actualidad conviven distintos tipos de programas de entrenamiento de memoria.

Será a partir de Israel (1988) que los programas se orientan a personas mayores sin deterioro cognitivo o adultas con quejas subjetivas de memoria. La efectividad de los programas y las buenas valoraciones por parte de los participantes facilitaron que se diseñaran intervenciones cognitivas para otros colectivos con necesidades distintas: niños, personas con discapacidad intelectual, con problemas de salud mental, etc.

Montejo (2015) define el entrenamiento de la memoria como «adiestrar de manera sistemática en el conocimiento, la utilización y el control de los procesos, estrategias, técnicas y vivencias implicadas en el funcionamiento de la memoria y en la mejora de su rendimiento» (p. 122).

En la actualidad, hay variabilidad en la conceptualización y estructuración de estos programas. Sin embargo, hay un cierto consenso en establecer que son propuestas de estimulación cognitiva dirigidas a personas sin deterioro cognitivo y que buscan optimizar el funcionamiento de su memoria con el fin de promocionar un envejecimiento activo (Díaz, Martín, \& Peraita, 2013).

En definitiva, los programas de entrenamiento de la memoria son intervenciones cognitivas, de carácter preventivo, dirigidas a personas adultas y mayores, fundamentadas en principios de neuroplasticidad cerebral con el fin de promocionar la reserva cognitiva, la capacidad de aprendizaje y la optimización de los distintos procesos cognitivos (Calero \& Navarro, 2006; Fernández-Ballesteros, 2009; García-Sevilla et al., 2014).

\subsection{Tipología de programas}

En la actualidad, hay una multiplicidad de programas de entrenamiento de la memoria. Difieren en objetivos, contenidos, metodología, duración y otros elementos de carácter organizativo (Vidal-Martí, 2016). Sin embargo, tienen en común tratar contenidos transversales como son: el entrenamiento de los dominios cognitivos, el conocimiento de la meta memoria, la utilización de las ayudas externas, las estrategias y técnicas para la memorización de los olvidos cotidianos (García-Sevilla et al., 2014). 
En la literatura, se establecen dos tipologías de programas de entrenamiento: los unifactoriales y los multifactoriales. Los programas unifactoriales fueron los primeros a diseñarse (Domínguez-Orozco, 2015).

Montejo, Montenegro, Reinoso, Andrés y Claver (2001) definen los programas unifactoriales como: «un tipo de método que estimulan un solo factor implicado en el proceso de la memoria lenguaje, atención, registro de la información, almacenamiento, recuperación, etc. - o bien una estrategia o técnica concreta» (p. 20).

Su finalidad es estimular un solo factor implicado o una estrategia para favorecer el entrenamiento de la memoria. Por ejemplo, entrenar el recuerdo de nombres, la asociación de caras y nombres, el recuerdo de textos u olvidos cotidianos son algunos modelos de este tipo de programa (Montenegro, 2016).

Yesavage, Sheikh, Friedman y Tanke (1990) fueron pioneros en diseñar un programa unifactorial de mayor trascendencia; consistía en trabajar el recuerdo cara- nombre mediante la estrategia de visualización. Sin embargo, dicho programa no es el único de esta tipología, sino que existen otros como Sanders y Sanders (1978), Rose y Yesavage (1983), Milders, Deelman y Berg (1998), Sanders y Sanders (1978), Sohlberg y Mateer (1987), Milders, Deelman y Berg (1998), Montenegro et al. (2001), Moreno y Lopera (2009), y García-Sevilla et al. (2014).

El segundo tipo de programas de entrenamiento son los programas multifactoriales; métodos de entrenamiento que trabajan procesos, estrategias y/o técnicas (Yesavage et al., 1990; Montejo et al., 2001; Hernández, 2005; Maroto, 2005; Fernández-Ballesteros, 2009; Gramunt, 2010). Su principal objetivo es influir en los diferentes factores implicados en el entrenamiento de la memoria (Ballesteros \& UNAM, 2002). Montenegro et al. (2001) establecen una taxonomía propia, diferenciando tres tipologías de programas: globales, modulares y centrados en los olvidos cotidianos.

Los programas globales, o también denominados integrales, entrenan procesos, estrategias y factores. Una de las características de dichos programas es su amplia aplicabilidad y el alto número de personas a las que se dirigen.

Los programas modulares se estructuran en módulos específicos, que parten de un análisis y de una planificación previa. Su particularidad es que, antes de su impartición, hay un trabajo previo que consiste en una detección de las necesidades, una priorización y un diseño de los módulos.

El tercer tipo de programas se centran en los olvidos cotidianos. Se orientan a contrarrestar olvidos producidos en la cotidianidad, en el día a día. Este tipo de programas, que son los menos utilizados, se focalizan en entrenar los olvidos más prototípicos de las personas en la cotidianidad, como por ejemplo los nombres de personas, de lugares... (Martín y Kaiser, 1998 (citado por García-Sevilla et al., 2014)).

Los programas multifactoriales de entrenamiento de la memoria se recomiendan más respecto a los unifactoriales (Pérez et al., 2015). Hay dos razones explicativas de dicha recomendación (Bäckman, 1990; Montejo Montenegro et al., 1999). Una primera es la optimización de los distintos procesos cognitivos; es decir, se trabajan todos los procesos, estrategias y técnicas y se facilita el establecimiento de nuevas relaciones neuronales, conllevando a una mayor eficacia de la función mental, la promoción de la reserva cognitiva y la cooperación de las diferentes funciones y procesos implicados (Montejo, Montenegro y Claver, 2002). Una segunda es la interrelación de las funciones cognitivas, condicionadas entre sí por los distintos factores del entorno, como por ejemplo las emociones, el interés y la motivación (Vidal-Martí, 2012a). Por consiguiente, estas dos diferencias reafirman que el entrenamiento multifactorial es más efectivo respecto al unifactorial.

\subsection{Metodología de intervención}

En la actualidad, hay dos tipos de metodología de intervención claramente diferenciadas: individual y grupal. El programa de entrenamiento de la memoria individual consiste en que la persona entrena la memoria de manera singular, siendo protagonista y único actor del proceso de entrenamiento. Hay distintos programas que posibilitan este tipo de entrenamiento y uno de los elementos de diferenciación es la factorialidad. En base a este último criterio, se dividen los programas en dos: unifactorialidad y multifactorialidad (Yesavage et al., 1990; Moreno \& Lopera, 2009; Gramunt, 2010; Vidal-Martí, 2015).

La segunda metodología de intervención es la grupal. Se fundamenta en el grupo: actividades realizadas, compartidas o evaluadas en grupo. Es decir, en el proceso de entrenamiento se proponen 
actividades para hacer en pequeño o en gran grupo; de manera individual pero analizada y comentada en pequeño grupo $y /$ o corregida en gran grupo.

$\mathrm{Al}$ igual que la metodología individual, el entrenamiento grupal puede ser de tipo unifactorial (Yesavage et al., 1990) o multifactorial (Israel, 1988; Montejo et al., 2001; Selva, 2002; Hernández, 2005; Maroto, 2005; Puig, 2007; Fernández-Ballesteros, 2009; Díaz, Martín, \& Peraita, 2013; Vidal-Martí, 2016).

Hay una tendencia a combinar las actividades individuales con las grupales. A esta combinación metodológica se denomina grupal, aunque se realice tanto trabajo individual como en grupo. La interacción entre los participantes facilita que las personas aprendan, se ayuden y, a su vez, puedan observar su progresión, comparándose y compartiendo las actividades con otros compañeros (VidalMartí y Pérez-Testor, 2016).

Algunos autores establecen que la metodología que combina el trabajo en grupo y el individual tiene mayores beneficios para la prevención del deterioro cognitivo que solo el individual. Los beneficios también son a nivel cognitivo, relacional y emocional (Vidal-Martí, 2012a; Montejo, 2015).

\subsection{Beneficios de los programas de entrenamiento grupal}

Distintos estudios demuestran la eficacia de los programas de entrenamiento de la memoria (Verhaeghen, Marcoen, \& Goosens, 1992; Stigsdotter-Neely \& Backman, 1995; Floyd y Scogin, 1997; Calero \& Navarro, 2006; Carrillo y Restrepo, 2009; Montejo, 2015). Sin embargo, el número de investigaciones centradas en los beneficios del entrenamiento en grupo es reducido. Novoa et al. (2008), en un meta análisis, establecen que las intervenciones en grupo tienen una mayor eficacia respecto a las individuales. Dichas investigaciones se centran en evaluar los aspectos cognitivos y no estudian las dimensiones relacionales y emocionales.

Toda actividad en grupo favorece la interrelación y la comunicación entre sus miembros. Cuando los participantes se encuentran en un espacio educativo que fomenta la relación, hay una mayor probabilidad para el diálogo y el intercambio. Una de las principales preocupaciones de los miembros es la pérdida de memoria. Si durante el programa se establece un espacio para compartir esta pérdida y se ofrecen conocimientos para entender el funcionamiento de la memoria a los participantes, la percepción de pérdida disminuye de manera notable (Vidal-Martí, 2012a).

La ayuda mutua es un segundo elemento relacionado con la dimensión relacional, que contribuye a un mayor beneficio del entrenamiento grupal. Las actividades grupales de tipo cooperativo facilitan el compañerismo, promueven la solidaridad, el apoyo, la colaboración y la unión entre sus participantes. Si se establecen espacios de comunicación, ya sean de carácter formal o informal, la ayuda mutua se intensifica e incrementa la motivación y el entrenamiento de los distintos dominios cognitivos.

El entrenamiento grupal es una fuente de aprendizaje. El trabajo en grupo aproxima a la persona a conocer otras maneras de hacer, actuar y vivir. Por consiguiente, el grupo es una fuente de conocimientos y experiencias. El entrenamiento en grupo facilita el aprendizaje de los contenidos propios del programa y los ocultos, más relacionados con las competencias relacionales y emocionales.

Un cuarto beneficio del entrenamiento grupal es el grado de satisfacción. Los participantes valoran el entrenamiento en grupo de manera más satisfactoria y positiva respecto el individual (Vidal-Martí \& Pérez-Testor, 2016). La metodología grupal posibilita la cooperación y la ayuda mutua; facilitando el aprendizaje de la meta memoria y las estrategias para resolver los olvidos cotidianos.

En definitiva, los programas de entrenamiento grupal contribuyen a una mejora del funcionamiento de la memoria, facilitan una mayor interrelación y comunicación entre sus participantes y permiten adquirir competencias comunicacionales, relacionales y emocionales.

\section{Bases del aprendizaje cooperativo}

Se entiende el aprendizaje cooperativo como una metodología educativa de carácter inclusivo que convierte la diferencia y la heterogeneidad como un elemento positivo de aprendizaje (Duran \& Monereo, 2012). Consiste en organizar el grupo- clase en pequeños grupos de trabajo, denominados equipos, estables en el tiempo, donde todos los participantes se convierten en agentes activos de su proceso de aprendizaje y se responsabilizan del propio proceso de aprender y de los compañeros de su equipo (García \& Rodríguez, 2010). El aula se convierte en un espacio de aprendizaje significativo; siendo el aprendizaje más duradero e integrativo (Slavin \& Johnson, 1999). 
El grupo es el motor del aprendizaje. Esto significa que el proceso de aprender se concibe como una actividad social, en que todos los participantes del pequeño grupo deben adquirir las competencias educativas trabajadas para conseguir el aprendizaje. Es una meta conjunta, un logro del trabajo en equipo; en definitiva, un producto de la actividad social entre sus participantes, dónde los más expertos ofrecen ayudas y soportes que facilitan al resto de participantes del equipo ser más competentes y autónomos (Wells, 2001 (citado por Duran y Monereo, 2012)).

La distribución de las tareas entre los participantes es un elemento crucial en el aprendizaje cooperativo. En las primeras sesiones, el profesor impulsará que cada participante exprese sus capacidades y dificultades en el equipo. La finalidad es ayudar a cada equipo a identificar sus potencialidades para contrarrestar las dificultades de los integrantes. La comunicación y la organización del equipo en base a la distribución de la tarea facilita la visualización de la diferencia como elemento positivo y motivador para la promoción del potencial educativo, facilitando que el equipo sea más que la suma de sus individualidades (Duran, Torró, \& Vilà, 2003).

Uno de los grandes beneficios del aprendizaje cooperativo es potenciar las habilidades psicosociales y de relación. Esta interacción se basa en tres valores principales: la ayuda mutua, el respeto y la solidaridad. A su vez, se fomenta el aprendizaje de habilidades relacionales como la aceptación de los distintos puntos de vista, la comunicación, la negociación, la promoción de la autoestima y la colaboración (Slavin, 1996).

Una de las funciones primordiales del profesor es estructurar las actividades con el fin de promocionar la comunicación e interacción de los participantes de manera positiva. A su vez, la estructuración facilita que cada participante asuma más responsabilidad individual y de equipo para conseguir el logro. La estructuración facilita que cada participante sepa que debe hacer a nivel individual y como miembro de un equipo.

El aprendizaje cooperativo se basa en el constructivismo. El conocimiento se construye, se crea en un proceso de interacción, donde el papel del profesor es mediar entre los participantes y los contenidos. La mediación entre iguales es más efectiva, porque la resolución y la gestión de los conflictos se realizan desde un mismo marco de experiencia cultural y lingüística, utilizando un mismo código y un vocabulario similar (Good \& Brophy, 1997 (citado por Duran \& Monereo, 2012)). Los participantes están más receptivos y sensibles a las dificultades de aprendizaje que puedan presentar sus compañeros de equipo y, por consiguiente, la ayuda mutua y el fomento de la cooperación es más auténtico.

Con la finalidad de promover un aprendizaje cooperativo, el profesor debe garantizar unas condiciones básicas, una estructuración educativa y organizativa y una metodología activa que favorezca la consecución de dicha metodología educativa (Kagan \& Kagan, 1994; Johnson \& Johnson, 2009; Duran \& Monereo, 2012; Peñalva \& Leiva, 2019). Estas condiciones son cinco: interdependencia positiva, responsabilidad individual y corresponsabilidad en grupo, el feedback y la retroalimentación del proceso evaluativo, las interacciones positivas y estimuladoras y el desarrollo de habilidades sociales.

Se entiende por interdependencia positiva la interacción entre los participantes, conllevando a que el logro individual este unido al logro del grupo y viceversa. Cada uno de los participantes depende del equipo y el equipo de cada uno de sus integrantes.

En el aprendizaje cooperativo cada uno de los participantes recibe retroalimentación de su proceso de aprendizaje por parte de los demás y del propio grupo. Este continuado proceso de feedback fomenta la responsabilidad individual y la corresponsabilidad del trabajo en equipo facilitando la consecución del logro (Ferreiro, 2007).

Las interacciones positivas y estimuladoras se conciben como el conjunto de relaciones abiertas, comprometidas e implicadas entre los miembros. Distintos autores (Johnson \& Johnson, 2009; Pujolàs, 2009; Duran \& Monereo, 2012) establecen la necesidad de limitar el número de participantes por equipo, con el fin de facilitar espacios de interacción y diálogo para tomar decisiones en grupo, consensuar objetivos y metas, resolver conflictos, crear espacios de confianza,...

Con el fin de promocionar un aprendizaje cooperativo, el profesor deberá introducir técnicas interpersonales y de equipo para estructurar la actividad y el proceso de aprendizaje. Duran y Monereo (2012) establecen que estas habilidades y técnicas son necesarias para fomentar la cooperación. Dedicar tiempo para aprender dichas técnicas en el aula es indispensable, con el objetivo que los participantes asimilen e integren dichas habilidades y se puedan implementar en el trabajo en equipo. 


\section{El aprendizaje cooperativo en el entrenamiento grupal y multifactorial}

El aprendizaje cooperativo como metodología de aprendizaje fomenta el logro del aprendizaje en equipo respecto a nivel individual. La implicación de todos los participantes en su respectivo equipo de trabajo facilita la consecución y el éxito. A su vez, permite el desarrollo de habilidades y estrategias relacionales, comunicativas y el fomento del trabajo en equipo. Esta metodología, a nuestro entender, es un buen método para aplicar en los programas de entrenamiento de la memoria.

A continuación, se presentan cuatro motivos que justifican porque se debe fomentar el aprendizaje cooperativo en los programas de entrenamiento grupal y multifactorial.

Un primer motivo es la participación. Los programas de entrenamiento grupal deben favorecer una participación basada en una interacción continuada en el tiempo, donde cada participante que asista pueda aportar sus conocimientos y compartir procesos, estrategias y factores de entrenamiento con los participantes de su equipo y con el profesional que dinamiza las sesiones.

El profesional que dinamiza las sesiones deberá crear un marco estructural, donde cada participante sea agente activo de su proceso de aprendizaje y de comunicación. La participación de todos los miembros del equipo debe garantizarse y será tanto el profesional como el propio equipo, quienes regulen y aseguren dicha participación.

La participación significa ponerse de acuerdo en todo aquello que hay que hacer y cómo se debe hacer. Es decir, el proceso de participación significa de manera implícita: consensuar, priorizar, decidir cómo se hace y distribuir tareas entre el equipo, realizar sus respectivas propuestas y valorar los resultados.

La implicación activa de cada uno de los participantes en su propio equipo facilita que el clima grupal propicie la acción, la interrelación, el aprendizaje y la movilización de la persona y de sus propios recursos personales. Es decir, una mayor implicación conlleva a una mayor motivación y participación por parte del participante en el programa de entrenamiento, favoreciendo la predisposición, la iniciativa, la motivación hacia el logro y probablemente un aumento de la comprensión y la calidad final de la actividad.

La implicación facilitará que se resuelvan las dudas y preguntas en relación al funcionamiento de la memoria entre los participantes del equipo y se den respuestas óptimas a las necesidades y demandas que las personas presentan durante el entrenamiento multifactorial.

Otro beneficio de la implicación es la visibilización de las necesidades reales de los participantes. La metodología cooperativa facilita que cada equipo visibilice los intereses de cada participante -un porcentaje importante de los cuales son comunes- y se establezcan las habilidades y estrategias para su resolución. El hecho de compartir necesidades empodera a sus participantes, facilita la capacitación de la persona para construir el equipo, refuerza la motivación grupal e individual y favorece el entrenamiento de la memoria durante el programa.

Un segundo motivo es el tipo de actividades. Las actividades deben ser significativas y dar respuesta a las necesidades de las personas, con el fin de fomentar su trasferencia a la vida cotidiana (Cuenca, 2011). La promoción de actividades significativas conlleva a una mayor implicación de los participantes y consecuentemente un incremento de la responsabilidad individual y corresponsabilidad grupal en el equipo.

Como establece Vigostky (1980), conectar con las necesidades de la persona facilita la comprensión, la relación y el aprendizaje. En definitiva, refuerza el binomio aprendizaje y desarrollo. Según este autor, si se parte de la zona de desarrollo próximo (ZDP) se promueve la motivación, el interés y consecuentemente el aprendizaje. Por consiguiente, si las actividades de memoria son significativas dan respuesta a las necesidades de los participantes, facilitan el proceso de comprensión, integración y aprendizaje, promueven la zona de desarrollo próximo y refuerzan los procesos, estrategias y factores para el entrenamiento de la memoria.

En los programas de entrenamiento de la memoria realizar actividades significativas posibilita un aprendizaje con sentido y responde a las necesidades de los participantes. Si estas actividades son en equipo refuerzan aún más el propio proceso de aprendizaje, contribuyen a desarrollar o mejorar las habilidades interpersonales y la consecución del logro. La metodología del aprendizaje cooperativo contribuye a reforzar los aprendizajes propios del entrenamiento y, a su vez, fomentar técnicas y habilidades interpersonales y de socialización basadas en la ayuda y colaboración mutua.

Los participantes mediante el aprendizaje cooperativo deben tener actitudes de implicación, de iniciativa, de obertura hacia los compañeros; y en relación a las tareas, las actitudes se deben orientar 
hacia a su resolución. Por consiguiente, la metodología educativa contribuye a intensificar el proceso de aprendizaje y romper atribuciones erróneas relacionadas con la edad de los participantes, como por ejemplo el mito de la incapacidad de aprender a una determinada edad.

El tercer motivo es la bidireccionalidad. Un elemento básico de un aprendizaje cooperativo es la bidireccionalidad; unas interacciones positivas y estimuladoras entre los participantes. A nuestro entender, los programas de entrenamiento de la memoria deben optimizar la reciprocidad, compartir las estrategias de memorización y todos aquellos conocimientos y habilidades que faciliten el aprendizaje de la propia memoria para su mejora. La bidireccionalidad rompe la barrera de quien sabe respecto de quien no sabe, fomentando el sentido y el porqué de la acción educativa.

La bidireccionalidad también puede contribuir a la mejora de la comunicación entre los participantes de los programas de entrenamiento de la memoria y optimizar la interdependencia positiva. Este tercer motivo es de suma importancia, porque favorece una mayor comunicación para facilitar la gestión de la ansiedad que causa la pérdida de memoria y reforzar el aprendizaje. Cuando se comparten y se refuerzan los conocimientos de la meta memoria, se ayuda al proceso de entrenamiento y se minimizan los efectos del proceso de introspección y de interioridad propio del proceso de envejecimiento.

El último motivo es la cooperación. La construcción del grupo y la ayuda mutua contribuyen al sentido de pertenencia, solidaridad y colaboración. Sentirse parte de un grupo es un factor de la cooperación. La cooperación facilita el desarrollo de habilidades y técnicas para el entrenamiento de la memoria y refuerza la motivación intrínseca (Panitz, 1999). Por consiguiente, las actividades de entrenamiento con la finalidad de fomentar la cooperación contribuyen al enriquecimiento, la ayuda mutua y el aprendizaje.

\section{Conclusiones}

Los profesionales de los programas de entrenamiento de la memoria tienen un nuevo reto: plantear una manera distinta de ayudar a aprender. El aprendizaje cooperativo contribuye a que los participantes aprendan, compartan, adquieran competencias relacionales y comunicativas mientras entrenan su memoria y, a su vez, encuentren respuestas a las dudas y necesidades de su entrenamiento.

Esta metodología contribuye a que los participantes se conviertan en agentes activos de su proceso de aprendizaje, fomentando la motivación, su implicación y participación; y promoviendo la adquisición de habilidades interpersonales y de equipo.

A su vez, la idoneidad del aprendizaje cooperativo como metodología favorecedora del proceso de enseñanza-aprendizaje en los programas de entrenamiento de la memoria se explica por cuatro elementos.

Un primero es la participación. La división de funciones y tareas en el equipo conlleva a que la carga de trabajo individual sea ligeramente inferior, conllevando a la promoción de aspectos relacionales y psicosociales del programa, situación que favorece el aprendizaje. La participación facilita que cada participante sea protagonista de su proceso de aprendizaje, facilitando la resolución de dudas y preguntas en relación a la memoria y el cómo facilitar el proceso de entrenamiento de tipo multifactorial.

A su vez, la participación favorece la posibilidad de conocer otras maneras de aprender. La intervención grupal y la metodología cooperativa contribuyen a que cada persona mayor pueda flexibilizarse, ampliar sus estrategias de memorización y por consiguiente aumentar su perspectiva. Esta posibilidad de incrementar conocimientos, estrategias y habilidades contribuirá a que la persona mayor pueda transferir las competencias adquiridas en el programa de tipo multifactorial a su cotidianidad.

Un segundo elemento es el sentido. Si se refuerza la metodología del aprendizaje cooperativo con las actividades significativas contribuirá al aprendizaje significativo, el aprendizaje con sentido. La persona mayor como participante del programa encontrará recursos y herramientas que podrá y deberá compartir. Este continuo intercambio en el seno del equipo más la participación, anteriormente comentada, facilitan la comprensión, la integración y el aprendizaje conllevando a reforzar las competencias relacionales y el entrenamiento multifactorial de la memoria.

Un tercer elemento es la interacción positiva y enriquecedora entre los participantes en el equipo. La interacción refuerza la participación activa de la persona, su implicación y su motivación hacia el logro. El aprendizaje cooperativo refuerza la implicación de la persona mayor en el programa de 
entrenamiento. La persona mayor, al adquirir competencias relacionales y de entrenamiento, podrá transferir el aprendizaje en su día a día.

Y un último elemento es su contribución a la cooperación. El aprendizaje cooperativo facilita que el participante, la persona mayor, sea agente activo, protagonista y se refuerce el sentimiento de utilidad y de ayuda mutua. Este refuerzo del sentimiento de utilidad y ayuda mutua en las personas mayores es un pilar para incrementar la autoestima y el propio autoconocimiento.

A lo largo de este artículo se analizaron y valoraron los beneficios del aprendizaje cooperativo. Sin embargo, es conveniente establecer cuáles son las limitaciones que presenta este artículo. Se destacan dos.

La metodología del aprendizaje cooperativo ha sido aplicada en contextos formales y de manera especial en niños. A nivel universitario con población adulta, hay investigaciones que abordan sus beneficios y su eficacia, pero son escasos los estudios existentes (Gómez \& Hernando, 2016; ValletBellmunt et al., 2017; Lanza \& Barrios, 2018).

La segunda limitación es la falta de implementación del aprendizaje cooperativo como metodología educativa en los programas de entrenamiento multifactorial.

A modo prospectivo, se recomienda implementar esta metodología de aprendizaje en los programas de entrenamiento de la memoria para conocer su real eficacia, validar los beneficios expuestos y confirmar que el aprendizaje cooperativo es una buena metodología para implementar en los programas de entrenamiento multifactorial y grupal de la memoria dirigidos a personas mayores.

\section{Referencias}

Bäckman, L. (1990). Plasticity of memory functioning in normal aging and Alzheimer's disease. Acta Neurologica Scandinavica, 82(S129), pp. 32-36. doi: 10.1111/j.1600-0404.1990.tb02602.x

Ballesteros, S., \& UMAM (2002). Aprendizaje y memoria. Madrid: UNED ediciones.

Calero, M. D., \& Navarro, E. (2006). La plasticidad cognitiva en la vejez: técnicas de evaluación e intervención. Barcelona: Octaedro.

Carrillo, C. M., \& Restrepo, F. L. (2009). Efectos de un entrenamiento cognitivo de la atención en el funcionamiento de la memoria de trabajo durante el envejecimiento. Acta Neurológica Colombiana, 25(4), pp. 244-251.

Cuenca, M. E. (2011). Motivación hacía el aprendizaje en las personas mayores más allá de los resultados y el rendimiento académico. Revista de Psicología y Educación, 6(1), pp. 239-254.

Díaz Mardomingo, M. C., Martín Quilis, Y. M., \& Peraita Adrados, H. (2013). Intervención cognitiva en personas sanas de la tercera edad (Un estudio piloto en Las Rozas de Madrid). Madrid: Editorial UNED.

Domínguez-Orozco, M. E. (2015). Entrenamiento para olvidos cotidianos y mejora de la memoria en mayores: resultados de efectividad. Pensando Psicología, 11(18), pp. 73-84. doi: 10.16925/pe.v11i18.1220

Duran, D., \& Monereo, C. (2012). Entramado: Métodos de aprendizaje cooperativo y colaborativo. Barcelona: Horsori Editorial.

Duran, D., Torró, J., \& Vilà, J. (2003). Tutoria entre iguals: un mètode d'aprenentatge cooperatiu per a la diversitat: de la teoria a la pràctica. Institut de Ciències de l'Educació, Universitat Autònoma de Barcelona.

Fernández-Ballesteros, R. (2009). Envejecimiento activo: Contribuciones de la psicología. Madrid: Pirámide.

Ferreiro Gravié, R. (2007). Una visión de conjunto a una de las alternativas educativas más impactante de los últimos años: El aprendizaje cooperativo. Revista Electrónica de Investigación Educativa, 9(2), pp. 1-9.

Floyd, M., \& Scogin, F. (1997). Effects of memory training on the subjective memory functioning and mental health of older adults: A meta-analysis. Psychology and Aging, 12(1), pp. 150. doi: 10.1037/0882-7974.12.1.150

García, A. J., \& Rodríguez, Y. T. (2010). Aprendizaje cooperativo en personas mayores universitarias. Estrategias de implementación en el Espacio Europeo de Educación Superior. Revista Interamericana de Educación de Adultos, 32(1), pp. 6-21.

García-Sevilla, J., Fernández, P. J., Fuentes, L. J., López, J. J., \& Moreno, M. J. (2014). Estudio comparativo de dos programas de entrenamiento de la memoria en personas mayores con quejas subjetivas de memoria: un análisis preliminar. Anales de Psicología, 30(1), pp. 337-345. doi: 10.6018/analesps.30.1.158021 
Gómez Pazos, M., \& Hernando Gómez, A. (2016). Experiencia docente acerca del uso didáctico del aprendizaje cooperativo y el trabajo de campo en el estudio del fenómeno de influencia social. Revista Electrónica Interuniversitaria de Formación del Profesorado, 19(2), pp. 331-346. doi: 10.6018/reifop.19.2.206921

Gramunt, N. (2010). Memoria y otros retos cotidianos. Vive el envejecimiento activo. Barcelona: Fundación La Caixa.

Hernández Viadel, J. C. (2005). Método UCLM para el entrenamiento de memoria en personas mayores. Tesis doctoral. Universidad de Castilla-La Mancha.

Israel, L. (1988). Método de entrenamiento de la memoria. Barcelona: Semar.

Johnson, D. W., \& Johnson, R. T. (2009). An educational psychology success story: Social interdependence theory and cooperative learning. Educational Researcher, 38(5), pp. 365-379. doi: 10.3102/0013189X09339057

Kagan, S., \& Kagan, M. (1994). Cooperative Learning. San Clemente.

Lanza Escobedo, D., \& Barrios Fernández, A. (2018). Aprendizaje cooperativo en el Espacio

Europeo de Educación Superior: influencia de la experiencia previa del alumnado en el desarrollo de competencias. CIDUI. Congrés Internacional de Docència Universitària i Innovació. Recuperado de https://www.cidui.org/revistacidui/index.php/cidui/article/view/123/111

Maroto, M. A. (2005). La memoria. Programa de estimulación y mantenimiento cognitivo. Madrid: Instituto de Salud Pública.

Milders, M., Deelman, B., \& Berg, I. (1998). Rehabilitation of memory for people's names. Memory, 6(1), pp. 21-36. doi: 10.1080/741941597

Montejo, P. (2015). Estudio de los resultados de un programa de entrenamiento de memoria y estimulación cognitiva para mayores de 65 años sin deterioro cognitivo. Tesis doctoral. Universidad Complutense de Madrid.

Montejo, P., Montenegro, M., \& Claver, M. D. (2002). Intervención en funciones cognitivas. Entrenamiento de memoria. Psiquiatría Geriátrica. Barcelona: Masson.

Montejo, P., Montenegro, M., Reinoso, A.I., de Andrés, M. E., \& Claver, M. D. (2001). Programa de memoria. Método UNAM. Madrid: Ayuntamiento de Madrid.

Montejo, P., Montenegro, M., Reinoso, A.I., de Andrés, M. E., \& Claver, M. D. (1999). Estudio de la eficacia de un programa de entrenamiento de memoria multicéntrico para mayores de 60 años. Revista Española de Geriatría y Gerontología, 34(4), pp. 199-208.

Montenegro, M., Montejo, P., Reinoso, A. I., \& de Andrés, M. E. (2001). Programas de entrenamiento de memoria. Método UMAM. Cuadernos de Trabajo Social, 14, pp. 255-278.

Montenegro Peña, M. (2016). Quejas subjetivas de memoria en el envejecimiento y en adultos jóvenes: variables implicadas. Tesis doctoral. Universidad Complutense de Madrid.

Moreno, C., \& Lopera, F. (2009). Efectos de un entrenamiento cognitivo de la atención en el funcionamiento de la memoria de trabajo durante el envejecimiento. Acta Neurológica Colombiana, 25(4), pp. 244-251.

Novoa, A. M., Juárez, O., \& Nebot, M. (2008). Efectividad de las intervenciones cognitivas en la prevención del deterioro de la memoria en las personas mayores sanas. Gaceta Sanitaria, 22(5), pp. 474-82. doi: $10.1157 / 13126930$

Panitz, T. (1999). Collaborative versus Cooperative Learning: A Comparison of the Two Concepts Which Will Help Us Understand the Underlying Nature of Interactive Learning. Recuperado de https://files.eric.ed.gov/fulltext/ED448443.pdf

Peñalva Vélez, A., \& Leiva Olivencia, J. J. (2019). Metodologías cooperativas y colaborativas en la formación del profesorado para la interculturalidad. Tendencias Pedagógicas, 33, pp. 37-46. doi: 10.15366/tp2019.33.003

Pérez, A., Roqué, M., Domènech, S., Monteserín, R., Soriano, N., Blancafort, X., Vidal, C., \& Gil, C. (2015). Efficacy of memory training in healthy community-dwelling older people: study protocol for a randomized controlled trial. BMC Geriatrics, 15(1), pp. 113. doi: 10.1186/s12877-015-01104

Puig, A. (2007). Ejercicios para mejorar la memoria. Madrid: CCS.

Pujolàs Maset, P. (2009). La calidad en los equipos de aprendizaje cooperativo. Algunas consideraciones para el cálculo del grado de cooperatividad. Revista de Educación, 349, pp. 225-239.

Sanders, R. E., \& Sanders, J. A. C. (1978). Long-term durability and transfer of enhanced conceptual performance in the elderly. Journal of Gerontology, 33(3), pp. 408-412. doi: 10.1093/geronj/33.3.408 
Selva, J. P. S. (2002). Entrenamiento en memoria autobiográfica sobre eventos positivos especificos en ancianos con sintomas depresivos. Tesis doctoral. Universidad de Castilla-La Mancha.

Slavin, R. E. (1996). Research on cooperative learning and achievement: What we know, what we need to know. Contemporary Educational Psychology, 21(1), pp. 43-69.

Slavin, R. E., \& Johnson, R. T. (1999). Aprendizaje cooperativo: teoría, investigación y práctica. Buenos Aires: Aique.

Stigsdotter-Neely, A. S., \& Backman, L. (1995). Effects of multifactorial memory training in old age: Generalizability across tasks and individuals. The Journals of Gerontology Series B: Psychological Sciences and Social Sciences, 50(3), pp. 134-140. doi: 10.1093/geronb/50B.3.P134

Sohlberg, M. M., \& Mateer, C. A. (1987). Effectiveness of an attention-training program. Journal of Clinical and Experimental Neuropsychology, 9(2), pp. 117-130. doi: 10.1080/01688638708405352

Rose, T. L., \& Yesavage, J. A. (1983). Differential effects of a list-learning mnemonic in three age groups. Gerontology, 29(5), pp. 293-298. doi: 10.1159/000213129

Vallet-Bellmunt, T., Rivera-Torres, P., Vallet-Bellmunt, I., \& Vallet-Belmunt, A. (2017). Aprendizaje cooperativo, aprendizaje percibido y rendimiento académico de la enseñanza de marketing. Educación XX1, 20(1), pp. 277-297. doi: 10.5944/educxx1.17512

Verhaeghen, P., Marcoen, A., \& Goossens, L. (1992). Improving memory performance in the aged through mnemonic training: a meta-analytic study. Psychology and Aging, 7, pp. 242-251. doi: 10.1037/0882-7974.7.2.242

Vidal-Martí, C. (2012a). Si entrenem, recordem. $2^{\mathrm{a}}$ edición. Barcelona: Claret.

Vidal-Martí, C. (2012b). Los talleres de entrenamiento de la memoria: un ejemplo de buena práctica para la promoción de la inclusión social en personas mayores. RES: Revista de Educación Social, 14. Recuperado de http://www.eduso.net/res/pdf/14/tame_res_\%2014.pdf

Vidal-Martí, C. (2015). Entrena tu memoria. 100 preguntas, 100 respuestas y 100 actividades. Barcelona: Invisibles.

Vidal-Martí, C. (2016). Actividades en grupo de mayores para entrenar la memoria. 60 propuestas de intervención. Madrid: CCS.

Vidal-Martí, C., \& Pérez-Testor, C. (2016). La evaluación de transferencia de un programa de entrenamiento de memoria. Aloma: Revista de Psicologia, Ciències de l'Educació i de l'Esport, 34(2), pp. 87-93.

Vidal-Martí, C., Vilar, R., Carrasco, O., Borrell, M., Capellades, A., \& García, P. (2013). Innovación en los talleres de entrenamiento de memoria. Educación Social. Revista de Intervención Socioeducativa, 54, pp. 153-163.

Vigotsky, L. S. (1980). El desarrollo pedagógico y psicológico. La Habana: Pueblo y Educación.

Yesavage, J. A., Sheikh, J. I., Friedman, L., \& Tanke, E. (1990). Learning mnemonics: Roles of aging and subtle cognitive impairment. Psychology and Aging, 5(1), pp. 133. doi: 10.1037/08827974.5.1.133 